Bronchiolitis is a common cause of respiratory illness in children resulting in significant pressures on health services. Hospitalisation occurs in up to $3.5 \%$ of cases and $10 \%$ of these will require admission to the paediatric intensive care unit (PICU).

Aim To review the patient characteristic's for all infants (less than 1 year old) who were admitted to the regional PICU requiring invasive ventilation for bronchiolitis, over the 10 year period from 1st January 2003 to 31st December 2012.

Method Data was retrospectively collected for all infants admitted to the regional PICU from PICANet data, the retrieval database and discharge letters were then reviewed. A range of data was collected including gestational age, age at presentation, presence of apnoeas, length of ventilation, length of stay, Respiratory syncytial virus status, use of inotropes and pre-existing diagnoses (particularly congenital heart disease and chronic lung disease).

Results 256 infants were invasively ventilated, there were 4 deaths (all had significant co-morbidities). 82\% of admissions were between November and January. There was a male predominance and two thirds of the infants ventilated were less than 2 months at presentation. The Mean length of PICU stay was 8 days and mean number of days ventilated $6.16 \%$ of patients required inotropic support. Interestingly $43 \%$ of infants with congenital heart disease who were ventilation for bronchiolitis required inotropes. As found by previous studies, apnoea was a common feature, this was associated with prematurity (64\% of infants $<32$ weeks, $65 \% \quad 32-37$ weeks, only $30 \%$ infants greater than 37 weeks).

Conclusion This study highlights high seasonal pressure on PICU of bronchiolitis and that apnoeas are a significant risk factor for PICU admission. It also shows that although bronchiolitis is commonly thought to be a single organ disease, it can have cardiovascular consequences.

\section{G387(P) ABSTRACT WITHDRAWN}

\section{G388(P) EARLIER EXTUBATION IS ASSOCIATED WITH A REDUCTION IN EARLY POST-OPERATIVE COMPLICATIONS IN CHILDREN UNDERGOING SURGERY FOR NON-IDIOPATHIC SPINAL DEFORMITY}

\footnotetext{
${ }^{1,2} \mathrm{CM}$ McDougall, ${ }^{1} \mathrm{H}$ McPherson, ${ }^{3} \mathrm{P}$ Cowie, ${ }^{1,3} \mathrm{~J}$ McFadzean. ${ }^{1}$ Paediatric Intensive Care, Royal Hospital for Sick Children, Edinburgh, UK; ${ }^{2}$ Paediatric Respiratory Medicine, Royal Hospital for Sick Children, Edinburgh, UK; ${ }^{3}$ Paediatric Anaesthesia, Royal Hospital for Sick Children, Edinburgh, UK
}

\subsection{6/archdischild-2015-308599.342}

Introduction and aims Surgical correction of non-idiopathic spinal deformities in children presents a challenge to intensivists because of the extensive nature of the surgery and co-morbidities of the patients. The postoperative management of such patients has not been standardised. Following a review of our practice, ${ }^{1}$ we have implemented a strategy of earlier extubation in those patients requiring post-operative ventilatory support, including increased use of non-invasive ventilation (NIV). This study aimed to assess the impact of this change in practice.

Methods All patients admitted to PICU following surgery for non-idiopathic spinal deformity were identified from the PICANet database for the time periods: (1) 1.1.07-31.12.08 ( $\mathrm{n}=64)$ and (2) 1.9.10-31.8.13 ( $\mathrm{n}=137)$. Details of the post-operative courses were obtained from the hospital casenotes and the PICU clinical information system.
Results The cohorts were similar with respect to age (mean (SD) $13.0(3.3)$ years $\mathrm{v} 12.8(4.2)$ years, $\mathrm{p}=0.70$ ), gender (male $52 \%$ $\mathrm{v} 47 \%, \mathrm{p}=0.77$ ) and proportion of patients with neuromuscular curve $(53 \% \mathrm{v} 46 \%, \mathrm{p}=0.43)$. A similar proportion received invasive ventilation (IV) in PICU $(42 \% \mathrm{v} 49 \%, \mathrm{p}=0.52)$ but the duration of IV was significantly shorter in the more recent cohort (median (IQR) 41(77.5) hours $\mathrm{v} 20(23)$ hours, $\mathrm{p}=$ 0.004). $11(17 \%)$ patients received NIV in cohort 1 compared to $45(33 \%)$ in cohort $2(\mathrm{p}=0.07)$. The total duration of respiratory support was reduced in cohort 2 but this did not reach statistical significance (median 4.0(8.2) v 2.4(4.6) days, $\mathrm{p}=0.20$ ). Postoperative complications occurred in 26(41\%) cases in cohort 1 and $37(27 \%)$ in cohort $2(\mathrm{p}=0.04)$, with a trend to reduced gastrointestinal complications in cohort $2(16 \% \mathrm{v} 9 \%$ patients, $\mathrm{p}$ $=0.11$ ). Median duration of post-operative hospital admission was 9(9) days in cohort 1 and 10(6) days in cohort $2(\mathrm{p}=$ $0.50)$.

Conclusions Early extubation, facilitated by use of NIV, is associated with a reduction in early post-operative complications. Other factors impacting the post-operative course require further study so that the pathway for these complex patients can be further refined.

\section{REFERENCE}

1 McDougall C, McFadzean J. Post-operative respiratory support following surgery for non-idiopathic spinal deformity in children. Pediatr Crit Care Med. 2011;12 (Suppl):A119

\section{G389(P) A CASE FOR EARLY AMMONIA TESTING IN ALL ENCEPHALOPATHIC PATIENTS: FEMALE PATIENTS WITH $X$-LINKED ORNITHINE TRANSCARBAMYLASE DEFICIENCY}

${ }^{1} \mathrm{C}$ Kanaris, ${ }^{2} \mathrm{~A}$ Ghosh, ${ }^{1} \mathrm{C}$ Partington. ${ }^{1}$ Paediatric Intensive Care Unit, Royal Manchester Children's Hospital, Manchester, UK; ' ${ }^{2}$ Department of Paediatric Metabolic Medicine, Royal Manchester Children's Hospital, Manchester, UK

\subsection{6/archdischild-2015-308599.343}

We report the case of a 14-year-old girl admitted to PICU following a four-day history of vomiting, unusual behaviour and progressive drowsiness. She had depressed consciousness and encephalopathic features, thus requiring intubation and ventilation. The initial CT and MR scans were unremarkable and an encephalopathy screen, including plasma ammonia was performed on admission.

Shortly after admission the patient developed seizures and signs of raised intracranial pressure with a right fixed, dilated pupil. The pre-ictal ammonia concentration was markedly elevated at $638 \mathrm{umol} / \mathrm{L}$. A repeat urgent CT brain showed diffuse cerebral oedema with signs of brain herniation; neuroprotective measures were therefore initiated. Attempts to rapidly reduce ammonia levels by haemofiltration and infusions of sodium benzoate and phenylbutyrate were biochemically successful. Despite a quick decline in ammonia levels the patient developed central diabetes insipidus and showed no signs of neurological recovery, with persistent fixed, dilated pupils. Biochemical investigations strongly suggested a diagnosis of ornithine transcarbamylase deficiency (OTCD) with low citrulline and increased urinary orotic acid. DNA for mutation analysis and a liver biopsy or enzyme studies were sent to confirm the diagnosis. Brain stem testing 5 days post- admission confirmed brain stem death.

OTCD is the commonest inborn error of the urea cycle and shows X-linked inheritance. The classic presentation in male hemizygotes is with life threatening hyperammonaemic coma 
shortly after birth but milder variants present later. The phenotype in female heterozygotes is variable and depends on the pattern of X-chromosome inactivation in hepatocytes. This can range from severe neonatal encephalopathy to being completely asymptomatic throughout life. Survival and neurocognitive outcomes can be better than in severely affected males but are variable. Aggressive treatment of hyperammonaemic crises, dietary management with low protein diets, and alternative pathway therapy prevent further cognitive decline.

This case highlights the importance of early ammonia testing in encephalopathic patients regardless of age. A diagnosis of OTCD should be considered in patients with hyperammonaemia regardless of sex. Early recognition and appropriate treatment are critical to avoid severe brain damage and death.

\section{G390(P) PICU OUTCOMES OF PAEDIATRIC ONCOLOGY PATIENTS IN A TERTIARY CENTRE}

L Prasad, A Pickard, R Thomas. Paediatric Intensive Care, Sheffield Childrens Hospital, Sheffield, UK

\subsection{6/archdischild-2015-308599.344}

Paediatric oncology patients form a significant proportion of patients admitted to PICU. We studied the short and long term survival of oncology patients admitted to a tertiary PICU.

Aim To evaluate the short-term and long -term survival of children admitted to PICU with cancer related problems and the impact of clinical variables on survival.

Methods Retrospective analysis of the PICANet data and case records of all oncology patients aged less than 16 years admitted to a tertiary PICU during a 5-year period from 2004 to 2008.

Results A total of 93 patients were identified, 90\% survived to discharge from PICU of which $58 \%$ survived to 5 years.

64 patients were admitted for post-operative care and 29 for medical reasons. All patients in the post-operative group survived PICU discharge while only 20 patients survived in the medical group; post-discharge mortality at 3 months, 2 years and 5 years was 5,12 , and 3 , and 5,5 , and 0 respectively.

\begin{tabular}{ll} 
Abstract G390(P) Table 1 & \\
\hline Type & Number \\
CNS tumour & 55 \\
Lymphoma & 4 \\
Leukaemia & 12 \\
Solid tumours & 15 \\
Others & 7 \\
\hline
\end{tabular}

Twenty patients received mechanical ventilation of which 7 survived; 13 received vasoactive drugs, of which 3 patients survived; 2 received renal replacement therapy but both died.

Nine patients had CNS complications (seizure/raised intracranial pressure), 2 died in PICU and 4 within 3 months of discharge.

Among PICU survivors the duration of stay was one day in $72 \%$; only $3(5 \%)$ received support for more than one organ system.

Among children who died on PICU, 6 died within 24 h; 7 (18\%) received support for more than one organ system.

Fifty eight per cent of patients with CNS tumours survived to 5 years. Only $37 \%$ of patients admitted with haematological malignancies survived to 5 years.
Conclusions PICU survival for patients admitted solely for postoperative care was better than those with medical complications.

Better survival was associated with fewer days in PICU and fewer organs supported.

The type of malignancy also had a significant impact on both short and long term survival after PICU admission.

\section{G391(P) USE OF NON-INVASIVE RESPIRATORY SUPPORT IN BRONCHIOLITIS: A NATIONAL SURVEY}

${ }^{1} \mathrm{H}$ Turnham, ${ }^{2} \mathrm{R}$ Agbeko, ${ }^{3} \mathrm{f}$ Furness, ${ }^{4} \mathrm{~A}$ Sutcliffe, ${ }^{1} \mathrm{P}$ Ramnarayan. ${ }^{1}$ Children Acute Transport Service, Great Ormond Street Hospital, London, UK; ${ }^{2}$ Paediatric Intensive Care, Great North Childrens Hospital, Newcastle-upon-Tyne, UK; ${ }^{3}$ Department of Paediatrics, Darlington Memorial Hospital, Darlington, UK; ${ }^{4}$ Institute of Child Health, London, UK

\subsection{6/archdischild-2015-308599.345}

Aims Bronchiolitis accounts for a significant proportion of hospital and intensive care admissions in children aged $<1$ year. ${ }^{1,2}$ Nasal continuous positive airway pressure (nCPAP) has been used traditionally as first-line respiratory support modality.

Heated-humidified High Flow Nasal Cannula (HFNC) therapy is rapidly gaining popularity as an alternate mode of noninvasive respiratory support due to its ease of use. This is despite lack of clinical trial evidence to support its use in bronchiolitis. ${ }^{3}$ We aimed to establish current practice relating to HFNC and CPAP use in the United Kingdom (UK).

Methods We devised an on-line survey (Survey Monkey, USA) and disseminated the link through regional retrieval services to hospitals with paediatric inpatient facilities. Responses were requested from one senior nurse and doctor from each hospital. The questionnaire covered: hospital characteristics, clinical indications for use of CPAP and/or HFNC in bronchiolitis, and willingness to participate in future research. Denominator data (number of UK hospitals with inpatient services) was obtained from the RCPCH 2013 census.

Results Five regional retrieval services distributed the survey to their local hospitals, covering 109 of 191 hospitals in the UK with inpatient paediatric services. 156 respondents from 102 individual hospitals provided information (response rate: 94\%).

Of the 102 hospitals, 23 had a dedicated paediatric intensive care unit, 38 a dedicated paediatric high dependency unit (PHDU), 50 had dedicated PHDU beds on general paediatric wards. 30\% have no PHDU provision. 95 (93\%) units can deliver nCPAP to children with bronchiolitis and 73 (71\%) can deliver HFNC. The main indications for HFNC use: as an alternative to nCPAP (69\%), escalation of therapy from low flow oxygen $(74 \%)$, and weaning from nCPAP $(57 \%)$. The majority (71\%) would choose HFNC over nCPAP in a deteriorating child with bronchiolitis; despite this, $76 \%$ were prepared to randomise infants between nCPAP and HFNC in a clinical trial.

Conclusions Use of HFNC is common across UK hospitals. Despite this, survey responses support clinical equipoise amongst the majority of clinicians, indicating the feasibility of a trial of nCPAP and HFNC at this time.

\section{REFERENCES}

1 Nagakumar P, Doull I. Current therapy for bronchiolitis. Arch Dis Child. 2012;97 (9):827-830

2 Paediatric Intensive Care Audit Network, 2013 Annual Report. Accessed June 2012

3 Beggs S, Wong ZH, Kaul S, Ogden KJ, Walters JA. High flow nasal canula therapy for infants with bronchiolitis. Cochrane Database Syst Rev 2014:CD009609 